\title{
MATHEMATICAL ANALYSIS OF COMBUSTION WAVES IN COMPETITIVE EXOTHERMIC REACTIONS
}

\author{
ZHEJUN HUANG
}

(Received 19 April 2018; first published online 19 July 2018)

2010 Mathematics subject classification: primary 35K55; secondary 35K57, 80A25.

Keywords and phrases: combustion waves, competitive exothermic reactions, reaction-diffusion system, stability analysis, bi-stability.

The phenomenon of combustion is undoubtedly one of the most important physiochemical processes in nature and industry. Combustion theory has played a significant role in the advancement of human civilisation and provided an understanding of natural phenomena such as energy production from burning fossil fuels, the synthesis of advanced materials and bushfires. Hence, there is a great value in understanding the process of combustion through mathematical modelling. The aim of this thesis is to investigate the properties and linear stability of combustionwave solutions of a reaction-diffusion system corresponding to two-step competitive exothermic reactions in a one-dimensional configuration. Such reactions have direct relevance to the combustion of $\mathrm{MeCH}_{2}$, where $\mathrm{Me}$ is either titanium or zirconium.

For the first part of this thesis, I begin by introducing the combustion model for twostep competitive exothermic reactions under adiabatic conditions and investigating the properties of adiabatic combustion waves. Shooting methods, relaxation methods, the method of lines and FlexPDE are employed to solve the governing equations. I find that, based on the definition of the crossover temperature, there are two different regimes determined by the choice of the parameters, including the ratios of the activation energies, pre-exponential factors and enthalpies [5]. The asymptotic flame speed and burnt temperature are obtained using the activation energy asymptotic method. The asymptotic results and numerical solutions are in good agreement.

Next, I show the existence of bi-stability where two different stable combustionwave solutions coexist for the same parameter values [3]. The fast- and slow-branch solutions can be obtained by choosing various ignition conditions. One solution can

Thesis submitted to the University of New South Wales in August 2017; degree approved on 28 November 2017; supervisors Harvinder Sidhu, Isaac Towers and Zlatko Jovanoski.

(C) 2018 Australian Mathematical Publishing Association Inc. 
switch rapidly to the other by changing the reactant temperature. The effects of the parameters used in the model on bi-stability are also investigated in this thesis.

I then consider an approximation of the Arrhenius reaction rate to simplify the combustion model for solid fuels and provide a phase-plane analysis [1]. By undertaking such an analysis, I am able to obtain an explicit form for the speed of the combustion wave. The asymptotic results show remarkable agreement with numerical solutions. In addition, the temperature and fuel mass fraction profiles are obtained using the piecewise-linear approximation.

For the final piece of work associated with the first part of this thesis, I use the Evans function method extended by the compound matrix method to study the linear stability of adiabatic combustion waves [6]. When bi-stability does not exist, the flame speed as a function of the activation energy is monotonic and the behaviour of the combustion waves is similar to that from the single-step reaction. When bi-stability exists, the flame speed as a function of the activation energy is s-shaped and the behaviour is much more complicated. I find that the fast branch is either completely stable or exhibits an oscillatory instability based on the existence of the AndronovHopf bifurcation point. On the slow branch, the behaviour is much more complicated and there are three scenarios: (i) the slow branch can be completely unstable starting from the turning point; (ii) there can exist only one Hopf point, so that the slow branch is first stable in a range from the turning point to the Hopf point and then loses stability; (iii) there can exist two Hopf points, so that the slow branch initially is unstable from the turning point to the first Hopf point, then becomes stable in the range from first to second Hopf points and then loses stability again.

For the second part of this thesis, I extend the previous work by taking heat loss into consideration. I begin by introducing the combustion model under nonadiabatic conditions [2]. I find that, as the heat-loss coefficient increases with other parameter values fixed, the flame speed decays gradually before the front ceases to exist. No propagating combustion waves exist when the parameter values of the activation energy and heat-loss coefficient are above the threshold values.

Next, I show that bi-stability also exists for the nonadiabatic case if the heatloss coefficient is below a certain threshold value [4]. Similar to the adiabatic case, the transition between the two stable branches can be observed by perturbing the temperature profile for the nonadiabatic case. Furthermore, including heat loss into the system has some 'stabilising' effect on the bi-stability behaviour. In other words, for the nonadiabatic case the solutions do not 'jump' from the slow branch to the fast branch unless a strong perturbation is applied to the system. Additionally, I present an investigation on the effect of heat loss on bi-stability [3]. I am able to investigate how heat loss affects the size of the bi-stable region using the method of lines.

I then consider an approximation of the Arrhenius reaction rate to simplify the combustion model for solid fuels under nonadiabatic conditions and provide a phaseplane analysis. I am able to obtain an explicit form for the speed of the nonadiabatic combustion wave. 
The final study in the second part of this thesis examines the linear stability of nonadiabatic combustion waves using a combination of the Evans function method and numerical simulation via the method of lines. When bi-stability does not exist, the flame speed as a function of the activation energy is c-shaped and the behaviour of the combustion waves is similar to that from the single-step reaction. When bi-stability exists, the flame speed as a function of the activation energy is $\mathrm{m}$-shaped and the behaviour is much more complicated. Compared with the adiabatic case, the inclusion of heat loss has a destabilising effect on the regions near the Hopf bifurcation points. In the bi-stable regions, there are two threshold values of the heat-loss coefficient: one, $l_{\mathrm{hp}}$, corresponds to the Hopf point; the other, $l_{\mathrm{e}}$, corresponds to the extinction point. When $l_{\mathrm{hp}}<l<l_{\mathrm{e}}$, transition to instability occurs and period-1 pulsating combustion waves are observed. When $l$ is increased further $\left(l<l_{\mathrm{e}}\right)$, more complicated oscillations are found.

To summarise, I have gained a better understanding of combustion waves for the two-step competitive exothermic reactions and am able to predict their behaviours. I also provide several prospects for further study as follows. It would be interesting to investigate the effect of the ambient temperature on the properties and linear stability of the combustion waves. The other possible extension is to investigate the threshold conditions for ignition as well as for regions where bi-stability was observed. The model considered in this thesis is based on two-step reaction chemistry in one spatial dimension. The investigation could be extended to consider models in two or three spatial dimensions. Finally, the approaches developed here for the competitive exothermic-reaction scheme can be extended to models with other combustion chemistry, such as sequential, parallel and chain-branching reactions. This would provide a better overall understanding of combustion processes.

\section{References}

[1] Z. Huang, H. S. Sidhu, I. N. Towers, Z. Jovanoski and V. V. Gubernov, 'Reaction waves in solid fuels for adiabatic competitive exothermic reactions', ANZIAM J(E) 56 (2015), C148-C162.

[2] Z. Huang, H. S. Sidhu, I. N. Towers, Z. Jovanoski and V. V. Gubernov, 'Investigating flame fronts in competitive exothermic reactions', in: MODSIM2015, 21st Int. Congr. Modelling and Simulation, Gold Coast, Australia, 2015 (Modelling and Simulation Society of Australia and New Zealand, Canberra, Australia, 2015), 64-70.

[3] Z. Huang, H. S. Sidhu, I. N. Towers, Z. Jovanoski and V. V. Gubernov, 'Nonadiabatic combustion waves in a two-step competitive exothermic-reaction model', ANZIAM J(E) 57 (2016), 14-31.

[4] Z. Huang, H. S. Sidhu, I. N. Towers, Z. Jovanoski and V. V. Gubernov, 'Analysis of bi-stability in combustion waves', in: Chemeca 2016, Chemical Engineering - Regeneration, Recovery and Reinvention, Adelaide, Australia (Engineers Australia, Melbourne, Australia, 2016), 242-251.

[5] Z. Huang, H. S. Sidhu, I. N. Towers, Z. Jovanoski and V. V. Gubernov, 'Properties of combustion waves in a model with competitive exothermic reactions', J. Math. Chem. 55(5) (2017), 1187-1201.

[6] Z. Huang, H. S. Sidhu, I. N. Towers, Z. Jovanoski and V. V. Gubernov, 'Stability analysis of combustion waves for competitive exothermic reactions using Evans function', Appl. Math. Model. 54 (2018), 347-360. 
ZHEJUN HUANG,

Applied and Industrial Mathematics (AIM) Research Group,

School of Physical, Environmental and Mathematical Sciences,

University of New South Wales at the Australian Defence Force Academy,

Canberra, Australia

e-mail: victor7hzj@sina.com 